\title{
Bladder hypocontractility-extending the indication for LDDM
}

\author{
Georgios Gakis, Milomir Ninkovic and Arnulf Stenzl
}

Since its clinical inauguration more than a decade ago, latissimus dorsi detrusor myoplasty (LDDM) has provided a new option for treating patients with impaired bladder contractility, who would otherwise have had to rely on lifelong clean intermittent catheterization (CIC). Dr Ginsberg's thoughtful comment on our long-term multicenter investigation with LDDM (Bladder acontractility: detrusor myoplasty and other options. Nat. Rev. Urol. 8, 185-186; 2011) ${ }^{1}$ raises some interesting aspects of our study that warrant further consideration.

Impaired bladder contractility owing to lower motor neuron lesion is a debilitating disorder, often affecting young patients. Tolerance of and adherence to CIC - the standard procedure for bladder emptying - ${ }^{2,3}$ is of the utmost importance to prevent functional deterioration of the lower and upper urinary tract. ${ }^{4}$ Nevertheless, even with strict adherence to CIC the risk of UTI, macrohematuria, urethral stricture and bladder stones cannot be ignored. For example, a prospective study that investigated 170 patients using CIC for neurogenic or non-neurogenic bladder dysfunction reported a 35\% rate of clinically apparent UTIs that substantially impaired well-being. ${ }^{5}$ However, it is certainly true that not all patients on CIC bear a psychological and socioeconomic burden. Some studies have noted an improvement in quality of life in $60 \%$ of patients, ${ }^{6}$ although the converse-the $40 \%$ who did not report a significant benefit-begs noting.

Patients using CIC often suffer from neurogenic bladder dysfunction owing to an upper motor neuron lesion that renders them inappropriate candidates for LDDM. But what of the minority of cases with hypocontractile bladder or bladder acontracility? Many of the patients in our study ${ }^{9}$ who opted for LDDM had performed CIC for several years and preoperatively reported a reduced quality of life. Over the long term, the integration of CIC into one's daily schedule can challenge both the patient and the physician who must guide treatment. In younger patients especially, resistance to a sickness role and the convenient permanent availability of materials to perform CIC several times a day can compromise their adherence to a sophisticated catheterization protocol. In older patients, physical and mental impairments can preclude the use of $\mathrm{CIC}$, leaving suprapubic catheterization as the only treatment option. ${ }^{7}$

Latissimus dorsi detrusor myoplasty was intended for a distinct group of patients suffering from an acontractile bladder and treatment-related adverse effects. ${ }^{8}$ Certainly, any surgical procedure restoring lower urinary tract function must prove its urodynamic safety over the long term. Nowadays, in light of recent data reporting long-term success rates of 70-80\%, LDDM is gaining more and more acceptance in the urological community as a surgical option to restore bladder contractility without endangering the upper urinary tract. ${ }^{9}$ However, owing to the complexity of the procedure and the rarity of the disease, LDDM is still performed only in referral centers. ${ }^{9}$

The cost-effectiveness comparison between life-long CIC and LDDM surgery is complex and depends on the reimbursement policy of each nation's health-care system. In a prospective randomized controlled trial, patients using hydrophilic catheters experienced less hematuria and withdrawal friction than those using uncoated catheters, but the hydrophilic catheters are associated with higher costs. ${ }^{2}$ In Germany, the current price for singleuse hydrophilic catheters is approximately $€ 3$ each, with a frequency of 4-5 times a day for a patient aged 40 years at initial diagnosis. This brings the projected lifetime sum (at 80 years) for single-use catheters to around $€ 300,000$ - approximately 15-fold higher than the total cost for LDDM surgery.
Predicting success in an individual patient opting to undergo LDDM surgery is as yet unfeasible owing to the low incidence of the underlying disease. From a statistical perspective, to provide a clear answer to this intriguing question one would have to identify independent parameters within multivariable analyses associated with improved outcome. In our long-term study of 24 patients treated in four different centers worldwide, it was not possible to preoperatively identify those parameters that indicated who would benefit most from surgery. A further study has examined the additional value that ambulatory urodynamic assessment might have in patients in whom conventional urodynamic assessment diagnosed acontractile bladder. ${ }^{10}$ The results revealed that some degree of bladder contractility was still present in $84 \%$ of patients, although micturition parameters such as postvoid residual urine volume were not significantly altered. ${ }^{10}$ Thus, one cannot exclude the possibility that a considerable number of patients in our study ${ }^{9}$ might have had detrusor hypocontractility rather than acontractility. Taking this into account, it seems reasonable to broaden the indications for LDDM to patients diagnosed with detrusor hypocontractility on conventional urodynamic assessment, who until now would not have been considered candidates. Not only might this be beneficial for a greater number of patients dependent upon lifelong CIC, but it might aid the accurate prediction of patients who will profit most from this challenging surgical procedure.

Department of Urology, University Hospital Tuebingen, Hoppe-Seyler Street 3, D-72076 Tuebingen, Germany (G. Gakis, A. Stenzl). Department of Plastic and Reconstructive Surgery, Hospital Munich-Bogenhausen, Englschalkinger Strasse 77, D-81925 Munich, Germany (M. Ninkovic). Correspondence to: G. Gakis georgios.gakis@web.de 
1. Ginsberg, D. A. Bladder acontractility. Detrusor myoplasty and other options. Nat. Rev. Urol. 8, 185-186 (2011).

2. Stensballe, J., Looms, D., Nielsen, P. N. \& Tvede, M. Hydrophilic-coated catheters for intermittent catheterisation reduce urethral micro trauma: a prospective, randomised, participant-blinded, crossover study of three different types of catheters. Eur. Urol. 48, 978-983 (2005).

3. Vapnek, J. M., Maynard, F. M. \& Kim, J. A prospective randomized trial of the LoFric hydrophilic coated catheter versus conventional plastic catheter for clean intermittent catheterization. J. Urol. 169, 994-998 (2003).
4. Ramm, D. \& Kane, R. A qualitative study exploring the emotional responses of female patients learning to perform clean intermittent self-catheterisation. J. Clin. Nurs. 20, 3152-3162 (2011).

5. Bakke, A. \& Malt, U. F. Psychological predictors of symptoms of urinary tract infection and bacteriuria in patients treated with clean intermittent catheterization: a prospective 7-year study. Eur. Urol. 34, 30-36 (1998).

6. Kessler, T. M., Ryu, G. \& Burkhard, F. C. Clean intermittent self-catheterization: a burden for the patient? Neurourol. Urodyn. 28, 18-21 (2009).

7. van Achterberg, T., Holleman, G., CobussenBoekhorst, H., Arts, R. \& Heesakkers, J.
Adherence to clean intermittent selfcatheterization procedures: determinants explored. J. Clin. Nurs. 17, 394-402 (2008).

8. Stenzl, A. et al. Restoration of voluntary emptying of the bladder by transplantation of innervated free skeletal muscle. Lancet 351 1483-1485 (1998).

9. Gakis, G. et al. Functional detrusor myoplasty for bladder acontractility: long-term results. J. Urol. 185, 593-599 (2011).

10. van Koeveringe, G. A., Rahnama'i, M. S. \& Berghmans, B. C. The additional value of ambulatory urodynamic measurements compared with conventional urodynamic measurements. BJU Int. 105, 508-513 (2010). 